\title{
Diversidade de Calliphoridae (Insecta: Diptera) na Base de Extração Petrolífera da Bacia do Rio Urucu, na Amazônia brasileira
}

\author{
Maria Cristina ESPOSITO¹, José Roberto Pereira de SOUSA², Fernando da Silva CARVALHO-FILHO ${ }^{3}$ \\ RESUMO \\ A diversidade e abundância de dípteros califorídeos de três ambientes (clareira artificial, clareira natural e mata) de Porto \\ Urucu/AM foram avaliadas em coletas anuais realizadas em 2004, 2005 e 2006. Ao longo destes três anos foram coletados \\ 2.121 exemplares pertencentes a 14 espécies. As espécies mais abundantes foram Chloroprocta idioidea (Robineau- Desvoidy), \\ Eumesembrinella randa (Walker) e Hemilucilia semidiaphana (Rondani). Os habitats de matas e clareiras naturais apresentaram \\ maior abundância de califorídeos quando comparados às clareiras artificiais, com índices de diversidade e equitabilidade \\ também maiores do que em clareiras artificiais, onde a dominância foi mais elevada.
}

PALAVRAS-CHAVE: Calliphoridae, moscas varejeiras, abundância, riqueza de espécies, Amazônia.

\section{Diversity of Calliphoridae flies (Insecta: Diptera) in the oil extraction field of Rio Urucu basin, in brasilian Amazonian}

\section{ABSTRACT}

The diversity and abundance of blow flies in three environments (anthropic gaps, tree-fall gaps, and primary "terra firme" forest) of Porto Urucu/AM were evaluated in annual collects realized in 2004, 2005, and 2006. During these three years were collected 2,121 specimens belonging to 14 species. The most abundant species were Chloroprocta idioidea (Robineau- Desvoidy), Eumesembrinella randa (Walker), and Hemilucilia semidiaphana (Rondani). Primary forests and tree-fall gaps enviroments, showed higher blow flies abundance than anthropic gaps, with diversity and equitability index higher than anthropic gaps, when the dominance value was high.

KEYWORDS: Calliphoridae, blow flies, abundance, richness, Amazon.

1 Universidade Federal do Pará - UFPA, E-mail: esposito@amazon.com.br

2 Universidade Federal do Pará - UFPA, E-mail: jrszoo@hotmail.com

${ }^{3}$ Universidade Federal do Pará - UFPA, E-mail: fernanbio@yahoo.com.br 


\section{INTRODUÇÃO}

As moscas são conhecidas por utilizarem matéria orgânica em decomposição para alimentaçấo e desenvolvimento de seus imaturos, desempenhando importante papel como organismos saprófagos. Vários graus de associações das moscas com o homem são encontrados, desde uma associação total, tornando-se dependentes de ambientes modificados pelo homem, até espécies que ocorrem apenas em ambientes naturais (Povolny, 1971). Essa sensibilidade em relação aos ambientes, aliada à rapidez de resposta em termos populacionais às modificaçôes antrópicas, confere às moscas a capacidade de funcionarem como indicadores de interferências humanas nos ambientes naturais (Esposito, 1999)

Tanto os trabalhos mais recentes como os das décadas de 70 e 80 revelam o predomínio das espécies invasoras de Chrysomya Robineau- Desvoidy, 1830 e sua interferência sobre as espécies nativas de Cochliomyia macellaria (Fabricius, 1775) e Lucilia eximia Wiedemann, 1819 na maioria dos ambientes amostrados das regiōes brasileiras (Baumgartner e Greenberg, 1984). No entanto, na Bacia do Alto Rio Urucu (Paraluppi, 1996) e na Floresta Nacional de Caxiuanã (Esposito, 1997, 1999), que são ambientes ainda bastante preservados da Amazônia foi constatado que não estavam colonizados pelas espécies invasoras de Chrysomya na década de 90 .

A regiāo da bacia do rio Urucu oferece uma oportunidade para o estudo das populaçóes de moscas em vários tipos de ambientes, inclusive os livres da influência humana, porque apresenta extensas áreas de florestas preservadas, bem como algumas áreas de clareiras que foram abertas para a prospecção de petróleo e gás natural pela Petrobrás S.A.

O objetivo deste estudo foi avaliar a diversidade e abundância de dípteros muscóideos da família Calliphoridae (Mesembrinellinae, Calliphorinae, Chrysomyinae) em três ambientes (clareira artificial, clareira natural e mata) de Porto Urucu/AM.

\section{MATERIAL E MÉTODOS}

O presente estudo foi desenvolvido na bacia do Rio Urucu, Amazonas ( $4^{\circ} 53^{\prime} 7^{\prime \prime}$; S 65 20'59” W), na área de exploração petrolífera da Petrobrás a $600 \mathrm{Km}$ oeste de Manaus. As coletas foram realizadas nos meses de setembro de 2004, junho de 2005 e junho de 2006, utilizando armadilhas com isca de 50 gramas de pulmáo bovino. As armadilhas são de fabricação caseira, similar a descrita por Almeida et al. (2003) e utilizada por Parallupi (1996) e Esposito (1997).

Em cada coleta foram expostas 120 armadilhas durante 48 horas. Nas duas primeiras coletas, três tipos de ambientes foram amostrados: 05 matas primárias, 05 clareiras artificiais (abertas para atividades da Petrobrás) e 05 clareiras naturais decorrentes principalmente da queda natural de árvores na mata. Na terceira coleta, dois tipos de ambientes foram amostrados: 15 clareiras artificiais e 15 matas.

As moscas foram preparadas e depositadas na coleção do Museu Paraense Emílio Goeldi (MPEG). A identificação dos dípteros foi realizada a partir das descriçóes e chaves de identificação de Guimarães (1977), Mariluis (1982), Peris e Mariluis (1984), Dear (1985), Toma (1995), Ribeiro e Carvalho (1998) e Carvalho e Ribeiro (2000). A subfamília Mesembrinellinae foi considerada como tal, apesar de Guimarães (1977) ter elevado este táxon para o nível de Família. Este critério foi adotado porque trabalhos de sistemática de Rognes (1986) e Toma e Carvalho (1995) continuam a considerá-la como um grupo interno á Calliphoridae.

Os índices de diversidade de Shannon-Weaner ( $\left.\mathrm{H}^{\prime}\right)$, equitabilidade (Shannon J') e dominância (Berger- Parker_D) foram utilizados para análise faunística dos três ambientes (clareiras artificiais, clareiras naturais e matas) (Magurran, 1988). Estes índices foram calculados pelo programa Species Diversity \& Richness 2.5 (Henderson e Seaby, 1997) com base nos dados das duas primeiras coletas.

\section{RESULTADOS E DISCUSSÃO}

A riqueza em Porto Urucu passou de sete espécies (Paraluppi, 1996) para um total de 14 espécies de califorídeos coletados neste trabalho, com o primeiro registro de Paralucilia adespota (Dear, 1985), Co. hominivorax, C. megacephala e as cinco espécies de mesembrinelíneos. Um total de 2.121 exemplares foi coletado e as espécies mais abundantes foram Chloroprocta idioidea (RobineauDesvoidy, 1830), Eumesembrinella randa (Walker, 1849) e Hemilucilia semidiaphana (Rondani, 1850). As espécies Cochliomyia hominivorax (Coquerel, 1858), Chrysomya megacephala (Fabricius, 1805) e Cochliomyia macellaria (Fabricius, 1775) foram representadas por apenas um individuo (Tabela 1).

Estudos sobre a fauna de moscas na Floresta Nacional de Caxiuanã, Melgaço, PA e matas na regiáo do Jarí, Monte Dourado, PA, verificaram também a predominância das mesmas espécies nativas Ch. idiodea, $H$. semidiaphana e $E$. randa (Esposito,1999; Esposito e Linhares 2002; Melo e Esposito 2006).

A espécie Ch. idioidea, que aparece em alta abundância, tem se mostrado um componente importante da fauna de califorídeos na região (Paraluppi, 1996). Já a coleta de apenas um indivíduo de Co. macellaria contrasta com os resultados anteriores, quando esta espécie representou 
cerca de $86 \%$ dos indivíduos coletados (Paraluppi, 1996), o que pode estar relacionado a uma possível alteração nas comunidades de Calliphoridae da regiâo. Vários trabalhos relatam drásticos decréscimos populacionais de Co. macellaria após a chegada das espécies exóticas de Chrysomya no Brasil (Guimaráes et al., 1979; Prado e Guimarães, 1982). No entanto, este náo parece ser o motivo da referida alteração, pois apenas quatro indivíduos do gênero Chrysomya foram coletados, demonstrando que, quase doze anos após o trabalho de Paraluppi (1996), a situação não se alterou em relação à colonização das espécies deste gênero na região.

Comparando os três ambientes com base nas duas primeiras coletas, as matas e clareiras naturais apresentaram abundância de califorídeos maiores do que as clareiras artificiais (Tabela 2 e Figura 1). Apesar das mesmas três espécies terem predominado nos diferentes ambientes (Figura 2 ), a espécie $E$. randa foi mais abundante nos ambientes de clareiras naturais e matas. Os mesembrinelineos de maneira geral apresentam hábito silvestre, vivendo em matas densas e úmidas (Peris e Mariluis, 1984).
A comunidade de califorídeos é mais diversa nas matas e clareiras naturais, pois estes ambientes apresentaram os maiores índices de diversidade e equitabilidade quando comparado às clareiras artificiais. Os índices de dominância das matas e clareiras naturais foram menores que o das clareiras artificiais (Figura 3). A tendência de redução na diversidade de califorídeos nos ambientes mais antropizados foi registrada por Esposito (1999) na Floresta Nacional de Caxiuanã, Melgaço, PA e no município de Portel, PA.

No entanto, percebe-se que há uma forte influência do ambiente de mata do entorno, tanto nas clareiras naturais quanto nas artificiais. Resultados semelhantes também foram obtidos entre dípteros fitosaprófitos e aves da regiáo, demonstrando que, apesar do forte impacto da produção das clareiras de poços e jazidas, a paisagem do Urucu ainda continua pouco alterada, com forte influência da mata primária (Aleixo et al., 2004).

Tabela 1 - Composição e abundância das espécies de Calliphoridae (Insecta, Diptera) coletadas na Província Petrolífera de Porto Urucu nos meses de setembro de 2004, junho de 2005 e junho de 2006.

\begin{tabular}{|c|c|c|}
\hline Subfamílias & Espécies & Total \\
\hline \multirow{8}{*}{ Chrysomyinae } & Chrysomya albiceps （Wiedemann, 1819) & 3 \\
\hline & Chrysomya megacephala (Fabricius, 1805) & 1 \\
\hline & Chloroprocta idioidea (Robineau-Desvoidy,1830) & 896 \\
\hline & Cochliomyia macellaria (Fabricius,1775) & 1 \\
\hline & Cochliomyia hominivorax (Coquerel,1858) & 1 \\
\hline & Hemilucilia semidiaphana (Rondani, 1850) & 272 \\
\hline & Hemilucilia segmentaria (Fabricius, 1805) & 8 \\
\hline & Paralucilia adesposta Dear, 1985 & 17 \\
\hline Calliphorinae & Lucilia eximia (Fabricius,1805) & 112 \\
\hline \multirow{5}{*}{ Mesembrinellinae } & Eumesembrinella randa (Walker, 1849) & 671 \\
\hline & Eumesembrinella quadrilineata (Fabricius, 1805) & 39 \\
\hline & Mesembrinella bellardiana (Séguy,1925) & 76 \\
\hline & Mesembrinella bicolor (Fabricius, 1805) & 2 \\
\hline & Mesembrinella batesi (Aldrich, 1922) & 22 \\
\hline Total & & 2.121 \\
\hline
\end{tabular}


Tabela 2 - Padrões de abundância das espécies de Calliphoridae (Insecta, Diptera) nos três ambientes (Clareiras artificiais-CLA, Clareiras naturais-CLN e Matas-MT) amostrados na Província Petrolífera de Porto Urucu nos meses de setembro de 2004 e junho de 2005.

\begin{tabular}{|c|c|c|c|c|}
\hline \multirow{2}{*}{ Espécies } & \multicolumn{4}{|c|}{ Ambientes } \\
\hline & CLA & CLN & MT & Total \\
\hline Chrysomya albiceps (Wiedemann, 1819) & 3 & 0 & 0 & 3 \\
\hline Chrysomya megacephala (Fabricius, 1805) & 0 & 0 & 1 & 1 \\
\hline Chloroprocta idioidea (Robineau-Desvoidy,1830) & 356 & 213 & 289 & 858 \\
\hline Cochliomyia macellaria (Fabricius,1775) & 1 & 0 & 0 & 1 \\
\hline Cochliomyia hominivorax (Coquerel,1858) & 0 & 0 & 1 & 1 \\
\hline Hemilucilia semidiaphana (Rondani, 1850) & 34 & 127 & 96 & 257 \\
\hline Hemilucilia segmentaria (Fabricius, 1805) & 2 & 1 & 5 & 8 \\
\hline Paralucilia adesposta Dear, 1985 & 10 & 1 & 6 & 17 \\
\hline Lucilia eximia (Fabricius,1805) & 4 & 6 & 8 & 18 \\
\hline Eumesembrinella randa (Walker, 1849) & 48 & 194 & 152 & 394 \\
\hline Eumesembrinella quadrilineata (Fabricius, 1805) & 3 & 17 & 5 & 25 \\
\hline Mesembrinella bellardiana (Séguy,1925) & 12 & 22 & 29 & 63 \\
\hline Mesembrinella bicolor (Fabricius, 1805) & 1 & 0 & 1 & 2 \\
\hline Mesembrinella batesi (Aldrich, 1922) & 1 & 5 & 14 & 20 \\
\hline Total & 475 & 586 & 607 & 1.668 \\
\hline
\end{tabular}

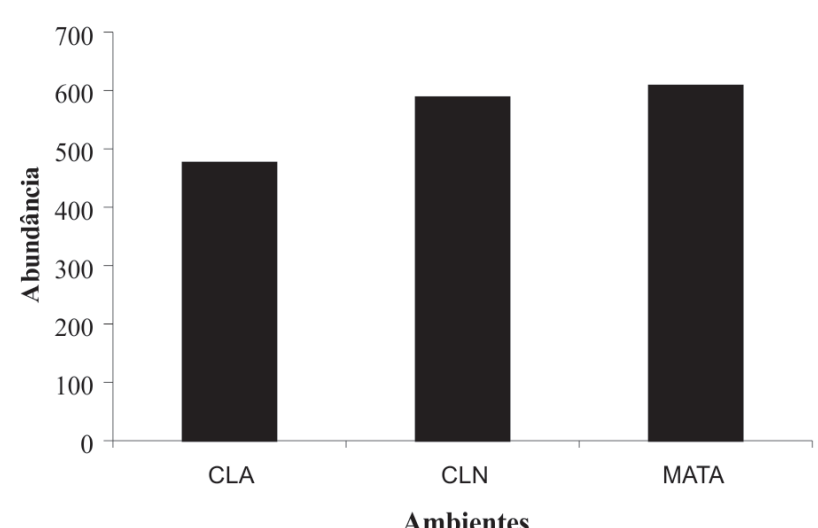

Figura 1 - Padrões de abundância de Calliphoridae (Insecta, Diptera) nos três ambientes (Clareiras artificiais - CLA, Clareiras naturais-CLN e Matas) amostrados da Província Petrolífera de Porto Urucu nos meses de setembro de 2004 e junho de 2005.

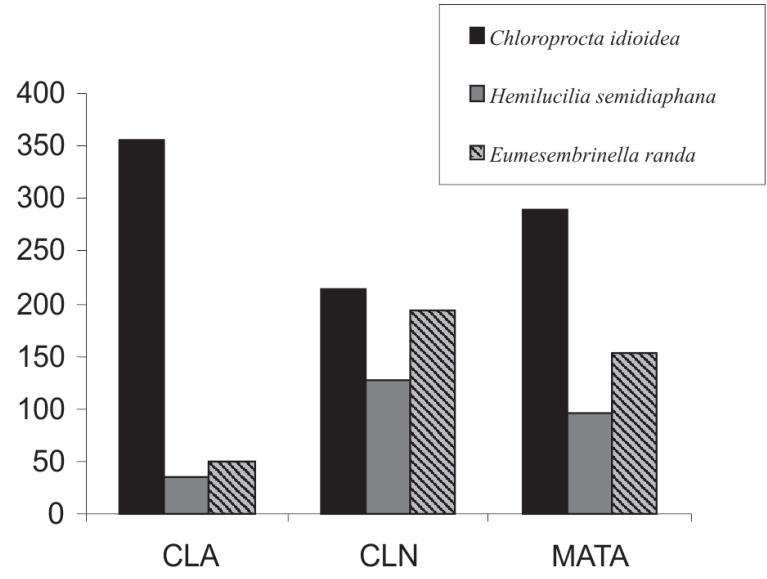

Figura 2 - Padrões de Abundância das espécies predominantes de Calliphoridae (Insecta, Diptera) nos três ambientes amostrados (Clareiras artificiais-CLA, Clareiras naturais-CLN e Matas) da Província Petrolífera de Urucu nos meses de setembro de 2004 e junho de 2005. 


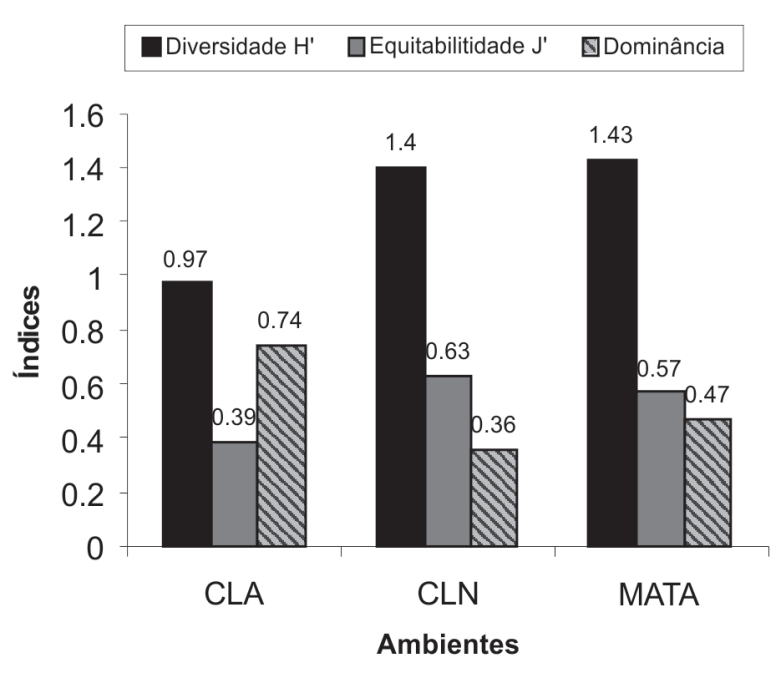

Figura 3 - Diversidade nos três ambientes amostrados (Clareiras artificiaisCLA, Clareiras naturais-CLN e Matas) da Província Petrolífera de Urucu nos meses de setembro de 2004 e junho de 2005.

\section{BIBLIOGRAFIA CITADA}

Aleixo, A.; Videira, M.L.M.; Poletto, F. 2004. Evaluation of fauna composition at gaps that will be recupered after oil exploration. In: I Workshop de Avaliação Técnica e Cientifica da rede CTPetro Amazônia. http://projetos.inpa.gov.br/ctpetro/Iworkshop/ pt1/pt1-02.pdf). Acesso:12/07/2007 (in Portuguese).

Almeida, L. M.; Ribeiro-Costa, C. S.; Marinoni, L. 2003. Collection, conservation, preparation and identification manual of insects. Série Manuais Práticos em Biologia-1. Ribeirão Preto, Holos Editora. 78 pp (in Portuguese)

Baumgartner, D. L.; Greenberg, B. 1984. The genus Chrysomya (Diptera: Calliphoridae) in the New World. Journal of Medical Entomology, 21(1): 105-113.

Carvalho, C.J.B.; Ribeiro, P. B. 2000. Identification key to Calliphoridae species (Diptera) in Southern Brazil. Revista Brasileira de Parasitologia Veterinária, 9 (2): 169-173 (in Portuguese, with abstract in English).

Dear, J. P. 1985. A revision of the New World Chrysomyini (Diptera: Calliphoridae). Revista Brasileira de Zoologia, 3: 109-169.

Esposito, M. C. 1997. The exotic species of Chrysomya (Diptera, Calliphoridae) in the Amazonia: Why they don't occur in the Ferreira Penna Cientific Station, p. 361-367. In: Lisboa P. L. B. (Ed.). Caxiuanã. CNPq-Museu Paraense Emílio Goeldi, Belém, Pará (in Portuguese).

Esposito, M. C. 1999. The blowflies fauna (Diptera, Calliphoridae) in the Amazon and their ecology at the Caxiuaná region and Portel City/ State of Pará.Tese de doutorado, Instituto Nacional de Pesquisas da Amazônia/Fundaçáo Universidade do Amazonas, Manaus, Amazonas, 134pp (in Portuguese)

Esposito, M. C.; Linhares, A.X. 2002. Calliphorids and other muscoids of the Ferreira Penna Cientific Station . p. 579-585. In: P. L. B. Lisboa (Ed.). Caxiauná. Tradicionals populations, Phisic conditions \& Biological DiversityCNPq-Museu Paraense Emílio Goeldi, Belém, Pará (in Portuguese).
Guimarães, J. H. 1977. A systematic revision of the Mesembrinellidae stat. nov. (Diptera, Cyclorrhapha). Arquivos de Zoologia (São Paulo), 29(1): 1-109.

Guimarães, J. H.; Prado A. P.; Buralli, G. M. 1979. Dispersal and distribution of three newly introduced species of Chrysomya Robineau-Desvoid in Brazil (Diptera, Calliphoridae). Revista Brasileira de Entomologia, 23:245-255.

Guimarães, J.H.; Papavero, N.; Prado, A.P. 1983. The myiases of Neotropical region (Identification, Biology and Bibliograph). Revista Brasileira de Entomologia, 1(4): 239-416.

Henderson, P. A.; Seaby, R. M. H. 1997. Species Diversity \& Richness v. 2.5. User Manual and Tutorial. Pennington \& Lymington, Pisces Conservation.

Magurran, A. E. 1988. Ecological Diversity and its measurement. Princeton University Press, Princeton, USA. 179pp.

Mariluis, J. C. 1982. Contribuition to the knowledge of Argentina Calliphoridae. (Insecta, Diptera). Opera Lilloana, 33: 1-58 (in Spanish with abstract in English).

Melo, A. G. D.; Esposito M. C. 2006 Abundance patterns and distribuition of muscoids flies in the Jari Celulose Forest, Monte Dourado, PA. p.191.In: Abstracts of the XVII Seminário de Iniciação Cientifica da Universidade Federal do Pará, Belém (in Portuguese)

Paraluppi, N. D. 1996. Calliphoridae (Diptera) in the Alto Urucu river basin, Central Amazonian, Brazil. Revista Brasileira de Zoologia, 13(3): 553-559 (in Portuguese with abstract in English)

Peris, S.V.; Mariluis, J.C. 1984. Notes on Mesembrinellidae. EOS - Revista Española de Entomologia, 60: 251-265 (in Portuguese with abstract in English).

Polvony, D. 1971. Synanthropy. In: Greenberg, B. (Ed.). Flies and Disease, Ecology, classification, and biotic associations, Vol. 1. Princeton Univ. Press, Princeton, N. J. p.17-54.

Prado, A.P.; Guimarães, J.H. 1982. Current state of the distribuition and dispersal of the species Chrysomya R-D genus in the Neotropical region (Diptera, Calliphoridae). Revista Brasileira de Entomologia, 26(3-4): 225-231 (in Portuguese with abstract in English).

Ribeiro, P. B. ; Carvalho, C.J.B. 1998. Pictorial Key to Calliphoridae Genera (Diptera) In Southern Brazil. Revista Brasileira de Parasitologia Veterinária, 7 (2): 137-140.

Rognes, K. 1986. The systematic position of the genus Helicobosca Bezzi with a discussion of the monophyly of the calyptrate families Calliphoridae, Rhinophoridae, Sarcophagidae and Tachinidae (Diptera). Entomologica Escandinavica, 17: 75-92.

Toma, R.; Carvalho, C.J.B. 1995. Filogenetic study of Mesembrinellinae with emphasis in the Eumesembrinella Townsend, 1931 (Diptera, Calliphoridae) genus. Revista Brasileira de Zoologia, 12: 127-144 (in Portuguese with abstract in English).

Recebido em 14/03/2008

Aceito em 31/07/2009 